\title{
Cardiovascular Responses to Feeding in Newborn Piglets
}

\author{
FRANK M. SCALZO \\ Division of Reproductive and Developmental Toxicology, National Center for Toxicological Research, Jefferson, \\ Arkansas 72079 and Department of Pediatrics, University of Arkansas for Medical Sciences and Arkansas \\ Children's Hospital, Little Rock, Arkansas 72205
}

\begin{abstract}
Cardiovascular responses to feeding have been observed in several species during various periods of development and have been implicated in the development of cardiovascular regulation. In the rat, these responses are characterized by short-lasting, large increases in blood pressure (BP) and moderate increases in heart rate. These responses appear to be sympathetically mediated because pretreatment with ganglionic blockers eliminates the increase in BP associated with milk ingestion. The present study was designed to determine if similar cardiovascular responses occur during feeding in the newborn piglet. Piglets were obtained on postnatal d 2 and fed a milk diet via automatic feeder 6 times a day. On postnatal d 6 , the piglets were instrumented with an external carotid artery catheter and an internal jugular vein catheter. On postnatal d 8 and 9, direct arterial $\mathrm{BP}$ and heart rate were recorded during feeding. BP responses to milk ingestion were immediate, and they reached a maximum increase of $50 \%$ above baseline on both test days and followed a response profile similar to that previously described in the 15-d-old rat. An increase in heart rate was also observed, reaching a maximum of $42 \%$ above baseline. The results show that early in life piglets have large cardiovascular respons $\omega_{j}$ to milk ingestion similar to those observed in young rats and human infants. These responses appear to model the cardiovascular responses to feeding observed in human infants and might be useful as a noninvasive method for assessing neonatal autonomic reactivity. These responses also have the potential to cause adverse effects in newborns already at risk for cardiovascular and cerebrovascular disease. (Pediatr Res 32: 33-38, 1992)
\end{abstract}

\section{Abbreviations}

BP, blood pressure

HR, heart rate

PND, postnatal day

ANOVA, analysis of variance

M1, milk-1

M2, milk-2

M3, milk-3

M4, milk-4

PH1, post hexamethonium-1

PH2, post hexamethonium-2

Received December 3, 1991; accepted February 25, 1992.

Correspondence: Frank M. Scalzo, Ph.D., Department of Pediatrics, MS-512B, University of Arkansas for Medical Sciences, 4301 W. Markham St., Little Rock, AR 72205.

Supported in part by an appointment to the Oak Ridge Associated Universities Postgraduate Research Program at the National Center for Toxicological Research administered by Oak Ridge Associated Universities through an interagency agreement between the U.S. Department of Energy and the U.S. Food and Drug Administration. The preparation of this manuscript was supported in part by NIDA Grant DA-06319.
Cardiovascular responses to feeding have been demonstrated in a number of species, including humans, during various periods of development (1-7). These responses typically consist of an abrupt BP increase and a moderate rise in HR. The mechanisms of these responses are not completely understood, but in the calf and rat pup they appear to involve neural-mediated vasoconstriction because they can be eliminated by ganglionic blockade with hexamethonium $(1,8)$. The biologic significance of these early cardiovascular responses to feeding is unclear. Recent studies have begun to address the notion that early feeding experience might play a role in the shaping of cardiovascular regulation (6, 8-11).

In young rats, cardiovascular responses to feeding appear to be an indicator of autonomic reactivity and might serve as an early indicator of cardiovascular systems predisposed to develop hypertension $(6,8)$. These responses appear to model the cardiovascular responses to feeding observed in human infants, in whom they might be useful as a noninvasive method for assessing neonatal autonomic reactivity $(2,3)$. The large excursions in BP and $\mathrm{HR}$ observed during the response might also have the potential to cause adverse effects in infants already at risk for cardiovascular or cerebrovascular disease.

The piglet was used in these studies in an attempt to develop an animal model of human infant cardiovascular responses to spontaneous feeding and to begin to address the hypothesis that under certain conditions (i.e. infants at risk for cardiovascular or cerebrovascular disease) large, feeding-induced excursions in $\mathrm{BP}$ and HR have the potential to cause adverse effects. The pig is well established as a model for studying human cardiovascular and digestive physiology (12-20). In fact, the piglet appears to be a better model than the infant rat for studying newborn feeding behavior and cardiovascular regulation. The piglet model allows for the analysis of physiologic variables not readily accessible in the infant rat (e.g. blood gases and hormones), and the infant piglet is easily reared away from the sow with artificial diets.

Houpt et al. (5) have reported BP and HR responses during spontaneous feeding in the young pig; however, only the infant rat has been intensively studied concerning the mechanisms and potential significance of cardiovascular responses to spontaneous feeding in newborns (6-8). In the present experiments, the newborn piglet ( 8 or $9 \mathrm{~d}$ old) was used as a model for human infant cardiovascular regulation. The goals of these studies were to develop methods for measuring cardiovascular responses to spontaneous milk ingestion in piglets, to determine if newborn piglets exhibit cardiovascular responses to spontaneous milk ingestion, and, if so, to characterize the responses and determine if they were sympathetically mediated as they have been previously reported to be in the calf and rat $(1,8)$. 


\section{MATERIALS AND METHODS}

All experiments were approved by the University of Arkansas for Medical Sciences Institutional Animal Use and Care Committee and were conducted in American Association for Accreditation of Laboratory Animal Care-approved facilities.

Subjects. Seven piglets were obtained on PND 2 from Cargill Swine Products (Russellville, AR). Piglets were housed in $1.0 \times$ $1.5 \mathrm{~m}$ Plexiglas cages with mesh floors made of rubber-coated metal. Temperature and humidity were maintained at $23^{\circ} \mathrm{C}( \pm$ $\left.3^{\circ} \mathrm{C}\right)$ and $50 \%( \pm 10 \%)$ in a room with a $12 \mathrm{~h}$ light-dark cycle (lights on at $0600 \mathrm{~h}$ ). Heat lamps were attached to the top of the cage and were directed into one corner of the cage. This placement allowed free access to the heated area. Piglets were fed an artificial milk diet (Land O'Lakes Littermilk w/PRT 99, Fort Dodge, IA) six times a day $(0200,0600,1000,1400,1800$, and $2200 \mathrm{~h}$ ) using an automated milk dispenser that delivered milk into a feeding trough $\left(15 \times 15 \mathrm{~cm}^{2}\right)$ that was attached to the home cage $7 \mathrm{~cm}$ above the floor and accessed through a hole 11 $\mathrm{cm}$ in diameter; water was available ad libitum. The manufacturer's recommendations for the volume and strength of the diet were followed, resulting in the delivery of six $250-\mathrm{mL}$ feedings of full-strength diet by PND 5. This feeding regimen resulted in a mean body weight of $3.0 \mathrm{~kg}( \pm 0.2$ SEM) on PND 8 , the first day of testing. Daily observations were recorded for body weight, rectal temperature, food intake, stools, and the general health and appearance of the animal.

Surgical procedures. On PND 6, piglets were anesthetized with a $2 \%$ isoflurane/oxygen mixture via spontaneous breathing. Under sterile conditions, a $5-\mathrm{cm}$ incision, $2 \mathrm{~cm}$ lateral to midline, was made to expose the carotid artery and jugular vein. The strap muscle (sternocephalicus) was resected, and the carotid artery was ligated at its rostral end and was clamped at its caudal end. A cut was made in the artery into which a length of Tygon tubing $(0.040$ inner diameter $\times 0.070$ outer diameter; Baxter Scientific, Grand Prairie, TX) filled with heparin $(10$ units $/ \mathrm{mL}$ saline) was inserted $2.5 \mathrm{~cm}$ into the artery to the tip of the aortic arch. The catheter was anchored to the rostral carotid ligature. The jugular vein was then cut, and a catheter, identical to the one used for the carotid, was inserted $2.5 \mathrm{~cm}$ into the vein and anchored to the rostral jugular ligature. The musculature and skin were closed with 3.0 sutures. The catheters were then tunneled s.c. to exit between the scapulas and were affixed to the skin with a suture. An injection cap was placed on the end of the jugular vein cannula, and a three-way stopcock closed the arterial catheter. Both catheters were wrapped in gauze and kept in place in with a polyester and rubber stretch bandage (MediTech International Corporation, Brooklyn, NY). After recovery, piglets were returned to their home cage, and the cannulas were flushed daily with heparinized saline (10 units/mL saline). Daily injections of antibiotic (trimethoprim-sulfadiazine $1.0 \mathrm{~mL} / 4.5$ $\mathrm{kg}$ body weight, s.c.) were given beginning on PND 6 and continued through PND 9.

\section{EXPERIMENT 1}

Procedure. On PND 8, the carotid cannula was connected to a pressure transducer for direct arterial pressure measurement using a polygraph (model 7, Grass Instrument Co., Quincy, MA). The BP signal was then calibrated with a mercury manometer, and both the carotid and jugular catheters were flushed with a heparinized saline solution (10 units/mL saline). The usual 250$\mathrm{mL}$ feeding at $1400 \mathrm{~h}$ was substituted by four $65-\mathrm{mL}$ feedings, M1, M2, M3, and M4, spaced 10 min apart. Milk was delivered into the feeding trough. This pattern of delivery was chosen because piglets had consumed all of the milk within 1-2 min in pilot studies. BP was monitored throughout the entire feeding period with milk dispensing times noted on the polygraph record.

Statistical analysis. Baseline BP and HR were calculated as the median value $5,10,15,20,25$, and $30 \mathrm{~s}$ before milk delivery. HR was estimated by counting the number of beats on the BP wave form for $5 \mathrm{~s}$ beginning at the points indicated. Median BP and $H R$ values were then averaged within groups to obtain group mean baseline values. BP and HR were determined at the onset of milk delivery $(0 \mathrm{~s})$ and $5,10,15,20,25,30,45$, and $60 \mathrm{~s}$ and $2,3,4,5,7$, and 10 min after milk delivery. Mean BP was calculated as the average of systolic and diastolic pressures, and mean HR was estimated by counting the number of beats on the $\mathrm{BP}$ wave form for $5 \mathrm{~s}$ beginning at the points indicated. ANOVA was used to analyze BP and HR data, and post hoc analyses were conducted using Duncan's multiple range test $(p<0.05)$.

\section{EXPERIMENT 2}

Procedure. The 2 nd experiment was conducted in an attempt to replicate the observations made on PND 8 and to determine if the cardiovascular response was sympathetically mediated as had been shown in the rat and calf $(1,8)$. On PND 9, the same procedure followed on PND 8 was repeated in six of the piglets, except that 10 min after the 3rd feeding (M3) the piglets were injected with $20 \mathrm{mg} / \mathrm{kg}$ hexamethonium (i.v.; Sigma Chemical Co.). The 4th (PH1) and 5th (PH2) feedings spaced 10 min apart were then given beginning $15 \mathrm{~min}$ after the injection.

Statistical analysis. The data from experiment 2 was analyzed in two stages. In the 1st stage, data scoring and analysis procedures similar to those used in experiment 1 were used to analyze the BP and HR responses for the first three feedings (M1-M3) on PND 9. In the 2nd stage, an analysis was conducted to assess the effects of hexamethonium on the $\mathrm{BP}$ and $\mathrm{HR}$ response.

\section{RESULTS}

\section{EXPERIMENT 1}

After preliminary analysis of the changes in BP and HR during milk ingestion, the maximum BP and HR values during each feeding were obtained and compared to baseline values to determine if there were significant differences between baseline and the maximum BP and HR value for each feeding.

Effects of milk ingestion on BP. A 4 (feeding) $\times 2$ (time) ANOVA with repeated measures across feeding and time was conducted to determine the effects of repeated feedings on baseline and maximum values reached during a 10 -min period after milk ingestion (Fig. 1). Piglets began consuming milk within seconds of delivery and typically consumed all of the diet within 1-2 min after delivery. A main effect of time $[F(1,6)=41.99, p$ $<0.01]$ and a feeding $\times$ time interaction $[F(3,18)=6.31, p<$ $0.01]$ were observed. Further analysis of the interaction revealed that baseline BP increased across feedings and was greater at the M4 delivery compared to M1 and M3 (Table 1). The maximum pressure reached decreased across feedings, but all increases above baseline were significant, with the largest change in BP observed on M1 and the smallest observed on M4 (Table 1).

The time to reach the maximum value did not differ across feedings although the rise time was fastest for M2. All peak increases were usually observed within the 1st min after milk delivery. A large amount of variability existed in the time for BP to return to baseline, ranging from just seconds after the peak change to several minutes. In some cases, BP did not return to baseline.

Effects of milk ingestion on $H R$. A 4 (feeding) $\times 2$ (time) ANOVA with repeated measures on feeding and time was conducted to determine the effects of repeated feedings on baseline and maximum HR values after milk ingestion (Fig. 2). A main effect of feeding $[F(1,18)=4.71, p<0.05]$ and time $[F(1,6)=$ $33.10, p<0.01]$ and a feeding $\times$ time interaction $[F(3,18)=$ $10.00, p<0.01]$ were observed. Further analysis of the interaction revealed that baseline HR increased after the 1 st feeding and then decreased thereafter to a level that was still greater than the M1 baseline (Table 1). The maximum HR reached decreased after the 2 nd feeding. All maximum increases were different 


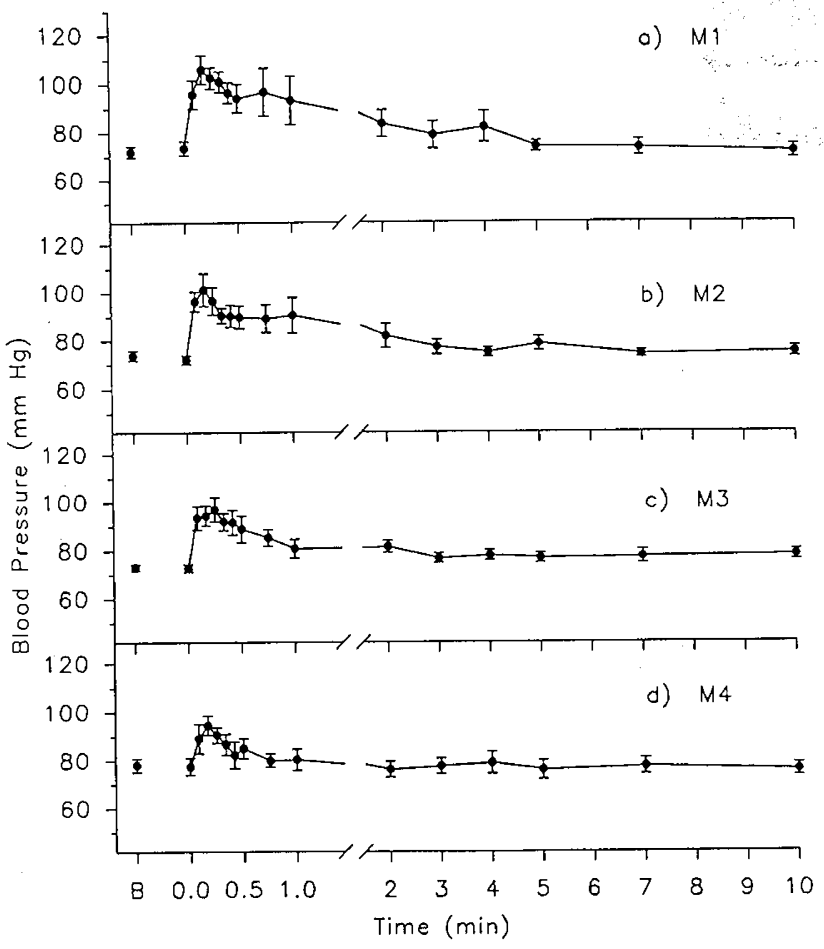

Fig. 1. BP (mean $\pm \mathrm{SEM}$ ) responses to the 1st ( $a, \mathrm{M} 1)$, 2nd (b, M2), 3rd $(c, \mathrm{M} 3)$, and 4 th $(d, \mathrm{M} 4)$ consecutive feedings on PND 8. Each point represents the mean of seven piglets. Points indicated are means during the following periods: baseline $(B, 0-30 \mathrm{~s}$ before milk delivery) and 0 (onset of milk delivery) $5,10,15,20,25,30,45$, and $60 \mathrm{~s}$ through 10 min after milk delivery.

Table 1. Baseline and maximum increases in $B P$ and $H R$ during feedings (mean $\pm S E M$ )

\begin{tabular}{lcccc}
\hline & M1 & M2 & M3 & M4 \\
\hline BP (mm Hg; $n=7)$ & & & & \\
Baseline & $72 \pm 2$ & $74 \pm 2$ & $73 \pm 1$ & $78 \pm 3^{*} \dagger$ \\
Maximum & $110 \pm 8 \ddagger$ & $104 \pm 6 \ddagger$ & $100 \pm 4 \ddagger$ & $97 \pm 5 \ddagger$ \\
HR (bpm; $n=7)$ & & & & \\
Baseline & $195 \pm 12$ & $230 \pm 10^{*}$ & $226 \pm 12^{*}$ & $216 \pm 7^{*}$ \\
Maximum & $276 \pm 8 \ddagger$ & $276 \pm 9 \ddagger$ & $265 \pm 10 \ddagger$ & $255 \pm 12 \ddagger$ \\
\hline
\end{tabular}

* Significantly different from $\mathrm{M} 1 ; p<0.05$.

$\dagger$ Significantly different from M3; $p<0.05$.

$\ddagger$ Significantly different from baseline; $p<0.05$.

from the respective baselines, with the largest change in HR observed on $\mathrm{M} 1$ and with the $2 \mathrm{nd}$, 3rd, and 4th feedings producing equal magnitudes of increase (Table 1 ).

The time to reach the maximum value did not differ across feedings. A large difference existed in the amount of time to return to baseline for $\mathrm{HR}$. On the average, $5 \mathrm{~min}$ elapsed before values returned to baseline after M1 compared to only $30 \mathrm{~s}$ after M2, M3, and M4.

\section{EXPERIMENT 2}

Effects of milk ingestion on BP. A 3 (feeding) $\times 2$ (time) ANOVA with repeated measures on feeding and time was conducted on the first three feedings (M1-M3) to determine the effects of repeated feeding on baseline and maximum BP values (Fig. 3). A main effect of time $[F(1,5)=43.52, p<0.01]$ and a feeding $\times$ time interaction $[F(2,10)=6.84, p<0.05]$ were observed. Analysis of the interaction revealed that baseline BP increased after the 1st feeding to reach equal baseline values for M2 and M3 (Table 2). The maximum pressure reached decreased across feedings, and all changes from baseline were significant,

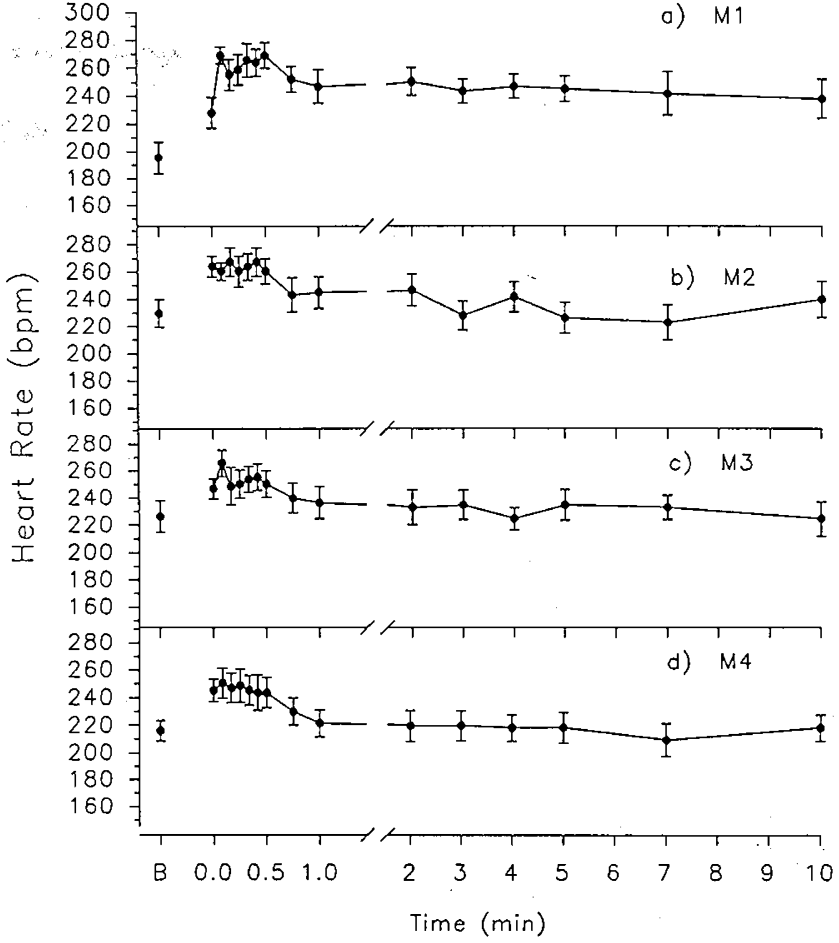

Fig. 2. HR (mean $\pm \mathrm{SEM}$ ) responses to the 1st ( $a$, M1), 2nd (b, M2), 3rd ( $c, \mathrm{M} 3)$, and 4th (d, M4) consecutive feedings on PND 8. Each point represents the mean of seven piglets. Mean HR for a given period was estimated by counting the number of beats on the BP wave form at the points indicated during the following periods: baseline $(B, 0-30 \mathrm{~s}$ before milk delivery) and 5-s periods beginning at 0 (onset of milk delivery), 5 , $10,15,20,25,30,45$, and $60 \mathrm{~s}$ through 10 min after milk delivery.

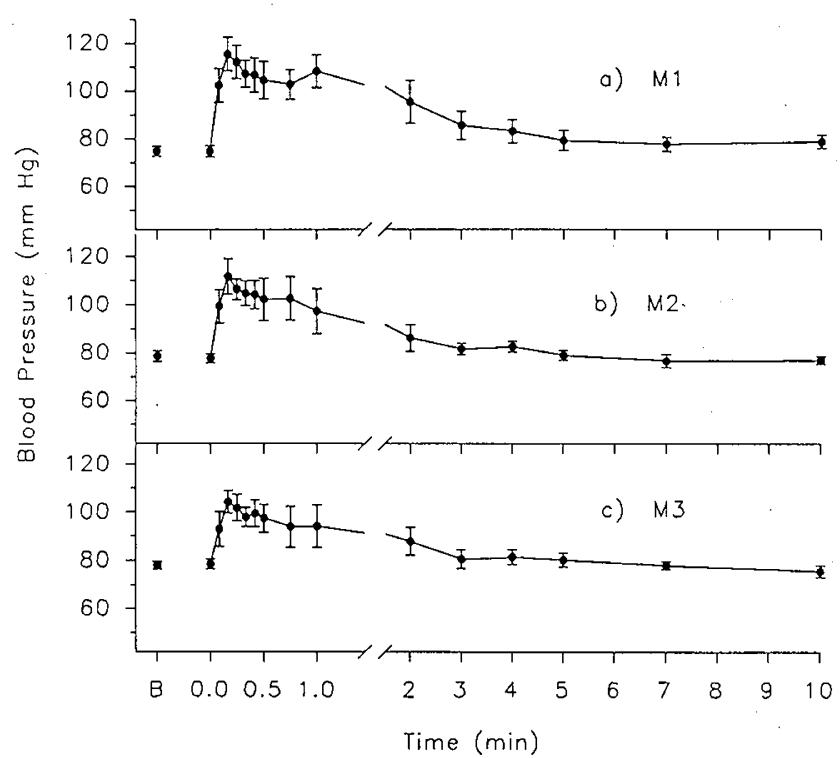

Fig. 3. $\mathrm{BP}$ (mean $\pm \mathrm{SEM}$ ) responses to three feedings ( $a, \mathrm{M} 1 ; b, \mathrm{M} 2$; and $c, \mathrm{M} 3$ ) on PND 9 before injection with hexamethonium (each point represents the mean of six piglets). Points indicated are means during the following periods: baseline $(B, 0-30 \mathrm{~s}$ before milk delivery) and 0 (onset of milk delivery), $5,10,15,20,25,30,45$, and $60 \mathrm{~s}$ through 10 min after milk delivery.

with the largest change in BP observed on M1 and the smallest observed on M3 (Table 2).

The time to reach the maximum value (14 s) did not differ across feedings. No differences in the time to return to baseline existed, and, as in experiment 1 , a large amount of variability in 
Table 2. Baseline BP and HR before feedings before and after treatment with hexamethonium $(20 \mathrm{mg} / \mathrm{kg}$, i.v.; mean \pm SEM) and maximum or minimum $B P$ and $H R$ during feedings before and after treatment with hexamethonium

\begin{tabular}{cccccc}
\hline & M1 & M2 & M3 & PH1 & PH2 \\
\hline BP (mm Hg; $n=6)$ & & & & & \\
Baseline & $75 \pm 2$ & $79 \pm 2^{*}$ & $78 \pm 2^{*}$ & $59 \pm 3 \dagger \ddagger$ & $59 \pm 4 \dagger \ddagger$ \\
Max or min & $115 \pm 7 \S$ & $113 \pm 7 \S$ & $108 \pm 6 \S$ & $46 \pm 4 \dagger \ddagger \S$ & $48 \pm 3 \dagger \ddagger \S$ \\
HR (bpm; $n=6)$ & & & & \\
Baseline & $184 \pm 16$ & $218 \pm 13^{*}$ & $218 \pm 9^{*}$ & $236 \pm 14$ & $226 \pm 12$ \\
Max or min & $270 \pm 9 \S$ & $278 \pm 9 \S$ & $262 \pm 9 \S$ & $220 \pm 13 \dagger \ddagger \S$ & $204 \pm 14 \dagger \ddagger \S$ \\
\hline
\end{tabular}

* Significantly different from M1; $p<0.05$.

$\dagger$ Significantly different from M2; $p<0.05$.

$¥$ Significantly different from M3; $p<0.05$.

$\S$ Significantly different from baseline; $p<0.05$.

this measure existed, with times ranging from just seconds after the peak change to several minutes. In some cases, BP did not return to baseline.

Effects of milk ingestion on $H R$. A 3 (feeding) $\times 2$ (time) ANOVA with repeated measures on feeding and time was conducted to determine the effects of repeated feedings on baseline and maximum HR values after milk ingestion (Fig. 4). Main effects of feeding $[F(2,10)=7.42, p<0.05]$ and time $[F(1,5)=$ 23.01, $p<0.01]$ and a feeding $\times$ time interaction $[F(2,10)=$ $4.54, p<0.05$ ] were found for HR. Analysis of the interaction revealed that baseline HR increased after the 1 st feeding to equal values for M2 and M3 (Table 2). HR increased to smaller maximum values on M2 and M3 compared to the highest maximum that was reached after the 1st feeding. All increases were significantly different from the respective baselines with the largest change in HR observed on M1 and M2; the increase for M3 was smaller than that on M1 (Table 2).

The time to reach the maximum value differed across feedings, with the larger increases taking longer to reach a maximum for $\mathrm{M} 1$ and M2. No differences existed in the amount of time to return to baseline, perhaps due to the large amount of variability in this measure. As in experiment 1 , the smaller responses returned to baseline faster.

Effects of hexamethonium on BP and HR responses to feeding. Based on the results thus far, it was apparent that the magnitude

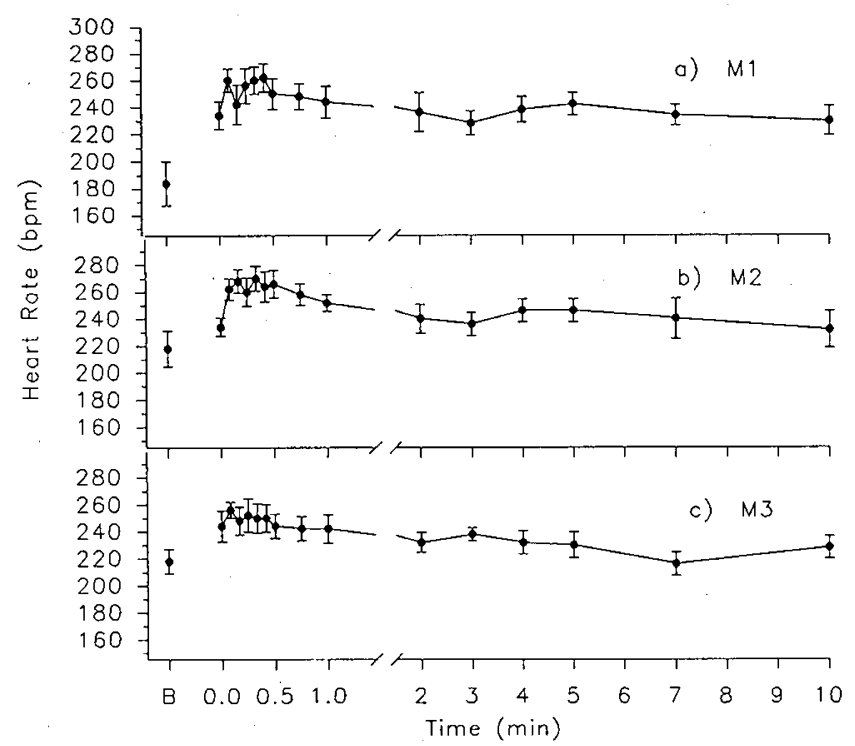

Fig. 4. HR (mean $\pm \mathrm{SEM}$ ) responses to three feedings ( $a, \mathrm{M} 1 ; b, \mathrm{M} 2$; and $c, \mathrm{M} 3$ ) on PND 9 before injection with hexamethonium (each point represents the mean of six piglets). Mean HR for a given period was estimated by counting the number of beats on the BP wave form at the points indicated during the following periods: baseline $(B, 0-30 \mathrm{~s}$ before milk delivery) and 5 -s periods beginning at 0 (onset of milk delivery), 5 , $10,15,20,25,30,45$, and $60 \mathrm{~s}$ through $10 \mathrm{~min}$ after milk delivery. and duration of the $\mathrm{BP}$ and $\mathrm{HR}$ response to M1 was different than the response to subsequent feedings. This 1 st feeding frequently produced an elevated baseline for HR and to a lesser extent for BP before the 2nd feeding. Because of this elevated baseline, it seemed more appropriate to compare the two feedings after injection with hexamethonium (PH1 and $\mathrm{PH} 2$ ) to the previous two feedings (M3 and M4). Therefore, a 2 (treatment) $\times 2$ (feeding) $\times 2$ (time) ANOVA with repeated measures on treatment, feeding, and time was conducted on BP and HR data to determine the effects of treatment with hexamethonium and repeated feedings on baseline and maximum $\mathrm{BP}$ and $\mathrm{HR}$ values after milk ingestion.

For BP, a main effect of treatment $[F(1,5)=60.62, p<0.01]$ and time $[F(1,5)=14.16, p<0.05]$ and a treatment $\times$ time interaction $[F(1,5)=59.02, p<0.01]$ were present. Analysis of the interaction revealed that BP increased above baseline after feeding before treatment with hexamethonium, whereas after hexamethonium treatment baseline BP decreased. Even though there were changes in BP after milk ingestion, these changes after hexamethonium were decreases compared to the increases observed in M2 and M3 (Fig. 5; Table 2). All changes from baseline were significant (Table 2). Because of the differences in baseline BP observed after hexamethonium, an analysis of the percentage of change of baseline was conducted. This analysis revealed a main effect of treatment $[F(1,5)=60.11, p<0.01]$ and a treatment $\times$ feeding interaction $[F(1,5)=7.45, p<0.05]$. The same pattern of results existed as was observed in the preliminary analysis; the percentage of change for the 2 nd feeding was smaller than the 1st feeding before and after treatment with hexamethonium. The percentage of change for M2 and M3 was an increase to $40 \%$ above baseline compared to a BP reduction to $80 \%$ of baseline on PH1 and PH2. The time to maximum change did not differ across feedings with both maximum increases and

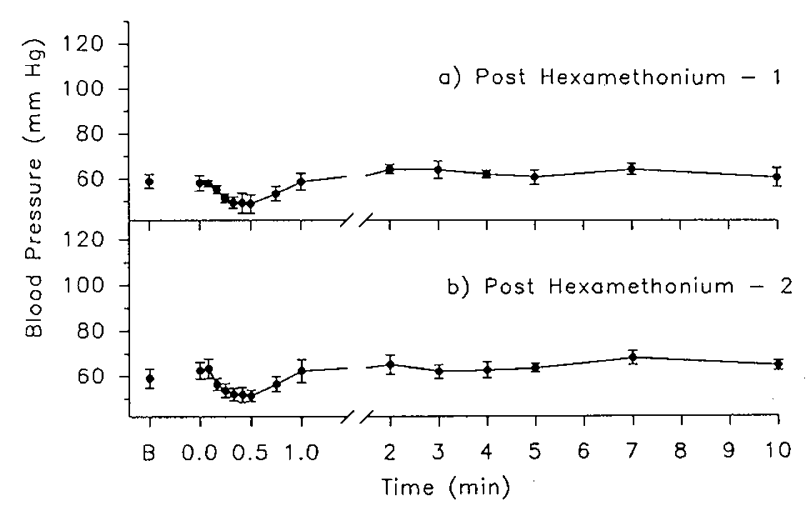

Fig. 5. $\mathrm{BP}$ (mean $\pm \mathrm{SEM}$ ) responses to two feedings ( $a$, $\mathrm{PH} 1 ; b, \mathrm{PH} 2)$ on PND 9 after injection with $20 \mathrm{mg} / \mathrm{kg}$ (i.v.) hexamethonium (each point represents the mean of six piglets). Points indicated are means during the following periods: baseline $(B, 0-30 \mathrm{~s}$ before milk delivery) and 0 (onset of milk delivery), $5,10,15,20,25,30,45$, and 60 s through 10 min after milk delivery. 
decreases taking 10-30 s. BP returned to baseline more quickly after treatment with hexamethonium.

For HR, a main effect of feeding $[F(1,5)=38.68, p<0.01]$ and a treatment $\times$ time interaction $[F(1,5)=44.3, p<0.01]$ were observed. Analysis of the interaction revealed that HR increased during feeding before hexamethonium treatment, whereas after hexamethonium treatment HR decreased during feeding. Baseline HR increased after hexamethonium treatment although this increase was not statistically significant compared to prehexamethonium values (Table 2). During $\mathrm{PH} 1$ and $\mathrm{PH} 2$, there were significant changes in HR after milk ingestion; however, these changes after hexamethonium were now decreases compared to the increases observed in M2 and M3 (Fig. 6; Table $2)$. The largest changes in HR were increases before hexamethonium treatment and were different from the 16-bpm decreases observed after hexamethonium treatment with the percentage of change before hexamethonium being an increase to $25 \%$ above baseline compared to a reduction in HR to $93 \%$ of baseline after hexamethonium.

The time to reach the maximum change did not differ across treatment or feeding, with both maximum increases and decreases taking approximately 5-15 s. No differences existed in the time to return to baseline for HR although there was a tendency for HR to return to baseline faster after hexamethonium treatment.

\section{DISCUSSION}

The results demonstrate that newborn piglets exhibit large increases in BP and HR during spontaneous feeding. The BP response reaches a maximum within seconds, with the increases reaching approximately $50 \%$ above baseline for the 1st feeding. The duration of the response is short, with values approaching baseline observed within 2-3 min after the onset of milk delivery. The magnitude of the response decreases with feedings. These results suggest that satiety factors may play a role in modulating the response, especially because by the 4th feeding both the magnitude of the response and the percentage of increase are less than those observed for the 1st feeding. In general, the HR response follows a similar pattern with two exceptions. First, the HR response appears to have an earlier onset, which may be due to cues associated with feeding (e.g. the sound of milk being dispensed and/or the sight of milk flowing into the feeding trough) that precede the actual ingestion of milk. Based on studies in the rat (6-8), the ingestion of milk is likely to have the direct effect of increasing BP. Second, baseline HR is increased after the 1 st feeding on both test days and remains elevated compared

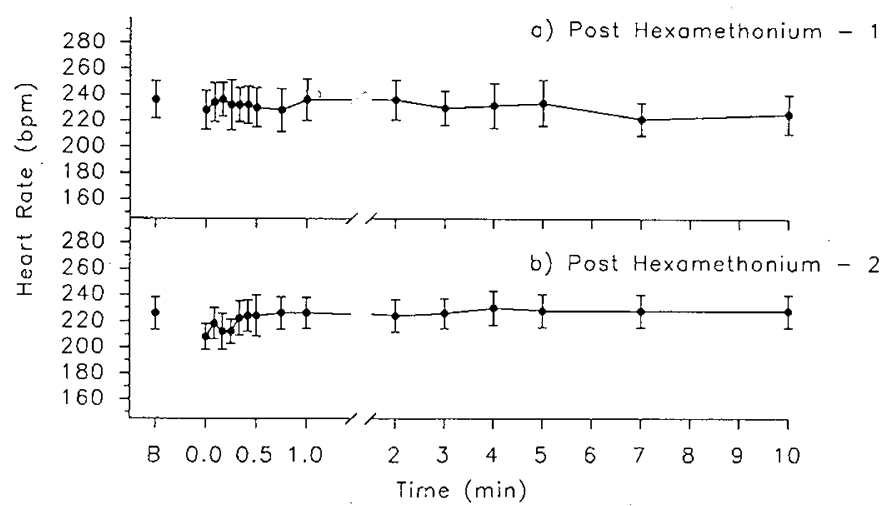

Fig. 6. HR (mean \pm SEM) responses to two feedings $(a) \mathrm{PH} 1$ and $(b)$ PH2 on PND 9 after injection with $20 \mathrm{mg} / \mathrm{kg}$ (i.v.) hexamethonium (each point represents the mean of six piglets). Mean HR for a given period was estimated by counting the number of beats on the BP wave form at the points indicated during the following periods: $\mathrm{B}$, baseline $(0-$ $30 \mathrm{~s}$ before milk delivery), 0 (onset of milk delivery), and $5,10,15,20$, $25,30,45$, and $60 \mathrm{~s}$ through $10 \mathrm{~min}$ after milk delivery. to the 1st feeding, whereas baseline BP did not consistently increase after the 1st feeding.

The characteristics of these responses appear to be stable both within and between test days. Within a day, both BP and HR responses appear to diminish with repeated feedings, although the smallest BP increases were still $30 \mathrm{~mm} \mathrm{Hg}$ or $38 \%$ above baseline and the smallest HR increases were $40 \mathrm{bpm}$ or $20 \%$ above baseline. The response does not appear to differ across test days, with similar patterns observed for the magnitude and duration of the response.

The BP and HR responses to feeding were eliminated by ganglionic blockade with hexamethonium. In fact, decreases in BP were observed during milk ingestion to approximately $80 \%$ of baseline, and HR decreased to $93 \%$ of baseline. It should be noted that these BP and HR decreases represent the largest change from baseline and that in some cases there were small, short-lasting increases in BP and/or HR (see Figs. 5 and 6), but the largest changes from baseline were indeed decreases for BP, whereas HR was only minimally affected. Treatment with hexamethonium also produced a reduction in baseline BP and elevated baseline $H R$ to a lesser extent, indicating that the dose administered was sufficient to produce at least a partial autonomic blockade.

The lack of a BP increase during feeding after pretreatment with hexamethonium suggests that sympathetic activation, which produces adrenergic vasoconstriction, mediates the BP response and that this activation, which also produces peripheral catecholamine release, contributes to the HR response. It is interesting to note that because the HR response had a faster onset than the BP response the HR response may involve a CNS component. There appeared to be a classically conditioned contribution to the HR response that might have produced the immediate $\mathrm{HR}$ increase as milk was being delivered into the feeding trough. Preliminary evidence from our laboratory suggests that this is indeed the case in young piglets, because presentation of the auditory and visual stimuli associated with milk delivery produces increases in $\mathrm{BP}$ and $\mathrm{HR}$ and the behavioral activation associated with feeding (Scalzo FM, unpublished observations). Harding et al. (4) reported that $\mathrm{BP}$ and $\mathrm{HR}$ increased in anticipation of feeding in lambs and then returned to or below baseline once suckling was initiated.

A remarkable similarity in the $\mathrm{BP}$ and $\mathrm{HR}$ responses to feeding was evident in the piglet compared to the responses observed in the human infant $(2,3)$, calf (1), older pig (5), lamb (4) and infant rat $(6,7)$. In general, all species exhibited an abrupt increase in BP and a smaller rise in HR. These responses appeared to be the largest changes in BP that were observed during several behaviors. In the present study, it was also observed that there were no changes in BP or HR during water intake from a feeding trough (Scalzo FM, unpublished observations).

The piglet thus appears to be a good model for further study of the mechanisms of these responses to spontaneous feeding. The piglet model has several advantages over the rat: piglets can easily be raised independent of the sow from an early age; they can be maintained on a variety of feeding schedules with various diet components; and several physiologic variables can be measured in piglets that are not readily accessible in the rat, such as endocrine and biochemical measures.

The ubiquity of the BP and HR responses to milk ingestion suggests that they might play a fundamental role in preparing animals for nutrient processing, perhaps by facilitating an increase in $\mathrm{O}_{2}$ supply to the gut. For example, Nowicki et al. (21) have shown increased $\mathrm{O}_{2}$ delivery to the gut effected by increased gastrointestinal blood flow during orogastric feeding in newborn piglets. Alternatively, because the $\mathrm{BP}$ and HR responses are elicited so frequently during early life, they could potentially be involved in shaping normal development of the cardiovascular system by establishing normal ranges for autonomic reactivity and maximum BP and HR. Although the responses have not been intensively studied in humans, comparable increases in BP 
and HR after breast-feeding and bottle-feeding have been reported in the human infant $(2,3)$. The large magnitude of the BP response adds an additional risk factor to cardiovascular systems already at risk, due to extreme genetic, congenital, or drug-induced levels of function.

In contrast, the lack of these repeated, large excursions in BP and HR might also have important implications for cardiovascular and gastrointestinal development. For example, human infants on total parenteral or enteral nutrition feeding regimens presumably do not experience these large BP and HR excursions. As a result, they might be at risk for cardiovascular or cerebrovascular pathologies when breast- or bottle-feeding is initiated. Similarly, because feeding-induced increases in BP and HR might play a role in the redistribution of blood flow to the gut in preparation for nutrient absorption, the lack of these increases might contribute to hypoxemia in the gut and place the neonate at risk for gastrointestinal complications (e.g. necrotizing enterocolitis).

Acknowledgments. The author thanks Theresa Sziszak and Tom Sziszak for technical assistance; Lora J. Burge, Margo Broening, and R. Robert Holson for assistance with data analysis; and Marjorie McMinn for editorial assistance. The support of Drs. Robert Warren and John Young is gratefully acknowledged.

\section{REFERENCES}

1. Bloom SR, Edwards AV, Hardy RN, Malinowska K, Silver M 1975 Cardiovascular and endocrine responses to feeding in the young calf. $J$ Physiol (Lond) 253:135-155

2. Cohen M, Fifer W, Better D, Dweck A, Myers M 1987 Elevation of blood pressure during feeding in newborns. Pediatr Res 21:383A(abstr)

3. Gupta JM, Scopes JW 1965 Observations on blood pressure in newborn infants. Arch Dis Child 40:637-644

4. Harding R, Johnson P, McClelland ME, McLeod CN, Whyte PL, Wilkinson AR 1978 Respiratory and cardiovascular responses to feeding in lambs. J Physiol (Lond) 275:40P-41P

5. Houpt KA, Baldwin BA, Houpt TR, Hills F 1983 Humoral and cardiovascular responses to feeding in pigs. Am J Physiol 244:R279-R284
6. Myers MM, Scalzo FM 1988 Blood pressure and heart rate responses of SHR and WKY pups during feeding. Physiol Behav 44:75-83

7. Shair HN, Brake SC, Hofer MA, Myers M 1986 Blood pressure responses to milk ejection in the young rat. Physiol Behav 37:171-176

8. Scalzo FM, Myers MM 1991 Pharmacological blockade of blood pressure and heart rate increases following milk ingestion in 15 day old SHR and WKY rat pups. Physiol Behav 50:525-531

9. Bell JM, Slotkin TA 1988 Postnatal nutritional status influences development of cardiac adrenergic receptor binding sites. Brain Res Bull 21:893-896

10. Bell JM, Whitmore WL, Slotkin TA 1988 Neonatal nutritional deprivation or enhancement: the cardiac-sympathetic axis and its role in cardiac growth and stress response. Pediatr Res 23:423-427

11. Myers M, Brunelli SA, Squire JM, Shindeldecker RD, Hofer MA 1989 Maternal behavior of the SHR rat and its relationship to offspring blood pressures. Dev Psychobiol 22:29-54

12. Bohr DF 1986 Hypertension studies in swine. In: Stanton HC, Mersmann HJ (eds) Swine in Cardiovascular Research. CRC Press, Boca Raton, FL, pp 157-176

13. Chiang SH, Pettigrew JE, Clarke SD, Cornelius SG 1989 Digestion and adsorption of fish oil by neonatal piglets. J Nutr 119:1741-1743

14. Dodds WJ 1990 The pig as a model for biomedical research. Fed Proc 41:247256

15. Douglas WR 1972 Of pigs and man in research: a review of administrations and analogies of the pig, sus scrofa, in human medical research. Space Life Sci 3:226-234

16. Flecknell P, Wootton A, Royston P, John M 1988 Glucose homeostasis in the newborn. Effects of oral feeding on response to fasting and intravenous glucose infusion in neonatal piglets. Biol Neonate 54:356-362

17. Gootman PM 1986 Development of central autonomic regulation of cardiovascular function. In: Gootman PM (ed) Developmental Neurobiology of the Autonomic Nervous System. Humana Press, Clifton, NJ, pp 279-326

18. Gootman PM, Gootman N, Turlapty PDMV, Yao AC, Buckley BJ, Altura BM 1981 Autonomic nervous system regulation of cardiovascular function in neonates. In: Elliot K, Lawrenson $\mathrm{G}$ (eds) Development of the Autonomic Nervous System. Pitman Medical, London, pp 70-93

19. Jones PJH, Hrboticky N, Hahn P, Innis SM 1990 Comparison of breastfeeding and formula feeding on intestinal and hepatic cholesterol metabolism in neonatal pigs. Am J Clin Nutr 51:979-984

20. Stanton HC 1986 Development of cardiovascular control and function. In: Stanton HC, Mersmann HJ (eds) Swine in Cardiovascular Research. CRC Press, Boca Raton, FL, pp 49-71

21. Nowicki PT, Stonestreet BS, Hansen NB, Yao AC, Oh W 1983 Gastrointestinal blood flow and oxygen consumption in awake newborn piglets: effect of feeding. Am J Physiol 245:G697-G702 